\title{
Newly synthesized palm esters for cosmetics industry.
}

\begin{abstract}
Palm esters were synthesized through enzymatic transesterification of various palm oil fractions with oleyl alcohol using Lipozyme RM IM as the catalyst. At the optimized alcoholysis reaction condition, after $5 \mathrm{~h}$ reaction time all palm oil fractions exhibited a high percentage yields of esters $(>80 \%)$. Simultaneous differential scanning calorimeter-thermal gravity analysis showed a high thermal stability profile of palm esters. Other physicochemical properties of palm esters such as refractive index, density, surface tension, slip melting point, saponification value, iodine value and acid value were analyzed following standard test methods modified from the American Oil Chemists' Society standards. The dermal irritation assay of palm oil esters shows the non-irritancy of the esters with a Human Irritancy Equivalent (HIE) score below 0.9. Furthermore, an increase in skin hydration of $40.7 \%$ after 90 min after application in an acute moisturizing test, has proven the suitably of palm oil esters to be used in the cosmetics formulation.
\end{abstract}

Keyword: Cosmetics ingredient; Moisturizing; Non-irritant; Palm esters; Physicochemical characteristics. 